\title{
Extraction and Separation Modeling of Orion Test Vehicles with ADAMS Simulation
}

\author{
Usbaldo Fraire Jr. ${ }^{1}$ \\ Jacobs Engineering, Houston, TX, 77058 \\ Keith Anderson ${ }^{2}$ \\ ATK Brigham City, Utah 84302 \\ P.A. Cuthbert ${ }^{3}$ \\ NASA-Johnson Space Center, Houston, TX, 77058
}

The Capsule Parachute Assembly System (CPAS) project has increased efforts to demonstrate the performance of fully integrated parachute systems at both higher dynamic pressures and in the presence of wake fields using a Parachute Compartment Drop Test Vehicle (PCDTV) and a Parachute Test Vehicle (PTV), respectively. Modeling the extraction and separation events has proven challenging and an understanding of the physics is required to reduce the risk of separation malfunctions. The need for extraction and separation modeling is critical to a successful CPAS test campaign. Current PTV-alone simulations, such as Decelerator System Simulation (DSS), require accurate initial conditions (ICs) drawn from a separation model. Automatic Dynamic Analysis of Mechanical Systems (ADAMS), a Commercial off the Shelf (COTS) tool, was employed to provide insight into the multi-body six degree of freedom (DOF) interaction between parachute test hardware and external and internal forces. Components of the model include a composite extraction parachute, primary vehicle (PTV or PCDTV), platform cradle, a release mechanism, aircraft ramp, and a programmer parachute with attach points. Independent aerodynamic forces were applied to the mated test vehicle/platform cradle and the separated test vehicle and platform cradle. The aero coefficients were determined from real time lookup tables which were functions of both angle of attack $(\alpha)$ and sideslip $(\beta)$. The atmospheric properties were also determined from a real time lookup table characteristic of the Yuma Proving Grounds (YPG) atmosphere relative to the planned test month. Representative geometries were constructed in ADAMS with measured mass properties generated for each independent vehicle. Derived smart separation parameters were included in ADAMS as sensors with defined pitch and pitch rate criteria used to refine inputs to analogous avionics systems for optimal separation conditions. Key design variables were dispersed in a Monte Carlo analysis to provide the maximum expected range of the state variables at programmer deployment to be used as ICs in DSS. Extensive comparisons were made with Decelerator System Simulation Application (DSSA) to validate the mated portion of the ADAMS extraction trajectory. Results of the comparisons improved the fidelity of ADAMS with a ramp pitch profile update from DSSA. Post-test reconstructions resulted in improvements to extraction parachute drag area knock-down factors, extraction line modeling, and the inclusion of ball-to-socket attachments used as a release mechanism on the PTV. Modeling of two Extraction parachutes was based on United States Air Force (USAF) tow test data and integrated into ADAMS for nominal and Monte Carlo trajectory assessments. Video overlay of ADAMS animations and actual C-12 chase plane test videos supported analysis and observation efforts of extraction and separation events. The COTS ADAMS simulation has been integrated with NASA based simulations to provide complete end to end trajectories with a focus on the extraction, separation, and programmer deployment sequence. The flexibility of modifying ADAMS inputs has proven useful for sensitivity studies and extraction/separation modeling efforts.

\footnotetext{
${ }^{1}$ Aerospace Engineer, Aeroscience and Flight Mechanics Section, 2224 Bay Area Blvd, Houston, TX, AIAA Member.

${ }^{2}$ Aerospace Engineer, Advanced Engineering, P.O. Box 707, Brigham City, UT.

${ }^{3}$ Aerospace Engineer, Aeroscience and Flight Mechanics Division, Houston, TX.
} 


\section{Nomenclature}

\begin{tabular}{|c|c|c|}
\hline A & $=$ & Airborne \\
\hline $\mathrm{A}_{\mathrm{ref}}$ & $=$ & Reference Area \\
\hline AESM & $=$ & ADAMS Extraction and Separation Model \\
\hline AGL & $=$ & Above Ground Level \\
\hline $\mathrm{C}_{\mathrm{x}}$ & $=$ & Force aerodynamic coefficient associated with the direction \\
\hline $\mathrm{C}_{\mathrm{xx}}$ & $=$ & Moment aerodynamic coefficient associated with the direction \\
\hline CDT & $=$ & Cluster Development Test \\
\hline $\mathrm{cm}$ & $=$ & Center of Mass \\
\hline $\mathrm{cp}$ & $=$ & Center of Pressure \\
\hline Downdraft & $=$ & Design variable with range of $2500 \pm 2000 \mathrm{lbs}$; dispersed during a Monte Carlo analysis. \\
\hline EDU & $=$ & Engineering Development Unit \\
\hline $\mathrm{Fa}$ & $=$ & Axial Drag \\
\hline Fn & $=$ & Normal, up and down \\
\hline Fy & $=$ & Side to side \\
\hline IMU & $=$ & Inertial Measurement Unit \\
\hline $\mathrm{L}_{\text {ref }}$ & $=$ & Reference Length \\
\hline Mll & $=$ & Moment about the roll axis \\
\hline $\mathrm{Mm}$ & $=$ & Moment about the pitch axis \\
\hline Mln & $=$ & Moment about the yaw axis \\
\hline $\bar{q}$ & $=$ & Dynamic Pressure \\
\hline Rho & $=$ & Atmospheric density. \\
\hline$C_{D} S_{r e f}(t)$ & $=$ & Extraction parachute apparent drag area as a function of time. \\
\hline $\mathrm{V}_{\mathrm{ex}}$ & $=$ & Velocity of the extraction parachute. \\
\hline $\mathrm{Vm}$ & $=$ & Magnitude of the extraction parachute velocity \\
\hline $\mathrm{V}_{\text {mated }}$ & $=$ & Velocity of the mated CPSS/PTV cg. \\
\hline $\mathrm{Vz}$ & $=$ & $\mathrm{z}$-component of the extraction parachute $\mathrm{cm}$ velocity vector \\
\hline
\end{tabular}

\section{Introduction}

$\mathrm{U}^{\mathrm{s}}$ NDERSTANDING the physics, dynamics and force interactions during extraction and separation events between an aircraft, test article, and platform are vital to the success of a Capsule Parachute Assembly System (CPAS) test campaign. Aerodynamics, atmosphere, vehicle configuration, mass properties, and the force interactions between these objects are key components that must be considered when designing for a drop test. Optimizing test vehicle mass properties to support end-to-end flight stability is critical, but a vehicle separation solution is necessary to initiate a favorable deployment sequence. Without a good separation solution, the CPAS drop test campaign would be at increased risk of loss-of-test-vehicle (LOTV) malfunctions during deployment.

The first test of a fully integrated CPAS system, Cluster Development Test 2 (CDT-2), occurred during the Generation I testing phase. The configuration consisted of a capsule shaped vehicle called a Parachute Test Vehicle (PTV) mated to a Cradle and Platform Separation System (CPSS). The approximately 30,000 lbs mated vehicle was extracted using a C-17 aircraft at an altitude of 25,000 ft. The CDT-2 test resulted in a LOTV due to a programmer deployment malfunction that occurred during the initial stages of flight after the PTV/CPSS separation event ${ }^{1}$. Valuable test experience was gained from this effort that is implemented in subsequent flights. To provide insight to the force interactions of test articles during the intricate extraction and separation flight phases, the development of the Commercial off the Shelf (COTS) tool Automatic Dynamic Analysis of Mechanical Systems (ADAMS) was employed. Efforts were invested to model the extraction-separation event of CPAS test articles based on physics principles versus qualitative solutions or assumptions.

Since the test execution of CDT-2, three successful Engineering Development Unit (EDU) capsule tests have been performed. EDU-A-CDT-3-3 was the first baseline capsule test after the CDT-2 mishap. The ADAMS Extraction and Separation Model (AESM) was used as the primary tool to provide nominal and Monte Carlo trajectories from aircraft extraction to PTV/CPSS separation through PTV programmer deployment. The state vector of the PTV in the AESM was delivered to Decelerator System Simulation (DSS) as initial conditions to provide predictions through vehicle touchdown. The preflight predictions were comparable to the flight test data. Post-test data reconstructions identified a delay in the cut command signal when the smart separation parameters 
were sensed by the avionics system. New release mechanism geometry was added to the AESM to account for the ball and socket interface interaction prior to a full PTV/CPSS separation.

The AESM gained increased fidelity for the second capsule test, EDU-A-CDT-3-5. The development of the release mechanism geometry simulated the contact forces with higher fidelity to give a better match of the separation delay seen in flight. An avionics system delay of $90 \mathrm{~ms}$ was also included in the smart separation logic to provide a representative cut command signal as seen in test. The test execution of EDU-A-CDT-3-3 and EDU-ACDT-3-5 proved the PTV $\backslash$ CPSS contact forces during separation consistently oriented the PTV heat shield forward at the release conditions despite the opposing aerodynamic moments that force the vehicle apex forward. The AESM simulations predicted this behavior prior to both tests. Post-Test data reconstructions introduced a downdraft force, due to the aircraft wake, on the extraction parachutes that resulted in a better match to test results. Each test experienced presumed downdraft forces and downdraft dispersion were included in the Monte Carlo analysis to account for the effects of this new parameter.

A conceptual three dimensional smart separation window was the answer to delivering preflight predictions with repeatable PTV heat shield forward attitudes. The three dimensions used to bound favorable PTV attitudes were time, pitch, and pitch rate. Each dimension was defined with an open and closed window criteria or minimum/maximum values. The introduction of an avionics time delay, release mechanism geometry and a downdraft force variable on the extraction parachutes lead to a family of solutions triggering at different sides of the defined smart separation window. Earlier preflight predictions only showed cases triggering at the maximum pitch rate window. Test results showed the mated vehicle sensing smart separation parameter at intended rates, but due to the time delay separated at lower rates than predicted. The inclusion of these parameters to the AESM proved successful during the third capsule test, EDU-A-CDT-3-7, which extracted from a C-17 and separated heat shield forward.

\section{Validating the AESM with DSSA}

Prior to integrating the ADAMS Extraction-Separation Model (AESM), into the CPAS mainstream simulation sequence, extensive trajectory comparisons were made using Decelerator Simulation System Application (DSSA) ${ }^{2}$. The pallet extraction simulation, DSSA, is a FORTRAN legacy simulation tool validated and used extensively for

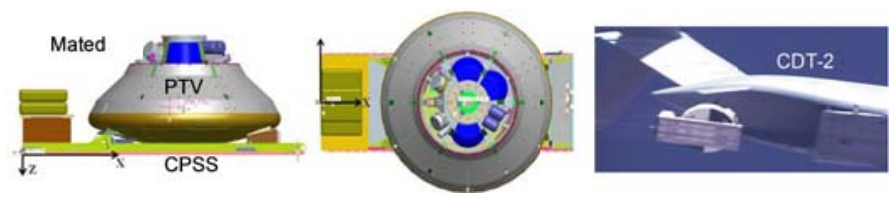
delivering preflight test predictions for CPAS in addition to the earlier NASA X-38 test program. DSSA is a variant of the DSS tool with added payload extraction capabilities. DSSA models two six degree-of-freedom (DOF) masses with

separate aerodynamic characteristics, coupled by an elastic riser - one body being the parachute, and the other the payload. Carrier aircraft ramp contact with the payload during extraction and tipoff pitch motions at ramp clear (RC) is also modeled. The simulation cannot track multiple independent bodies after the separation event, such as a PTV/CPSS would demonstrate in flight. Thus, it is only used for test configurations that include a single non-separating vehicle configuration such as a weight tub on a pallet for CPAS, and/or a Crew Return Vehicle (CRV) for X-38. Early hand-off conditions were generated using the mated portion of flight at selected times with favorable pitch and pitch rate dynamics. The crude separation hand-off condition was assumed to be instantaneous. Due to DSSAs proven test heritage and fidelity it was used to verify and validate
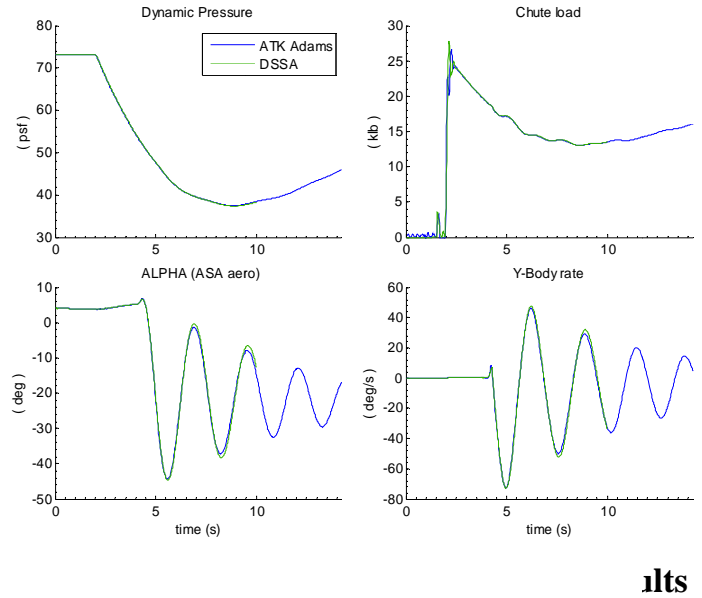
preliminary trajectories produced by the AESM.

The analysis in this section was performed prior to the EDU-A-CDT-3-3 test execution. The intent of this analysis was to compare AESM results to the mated portion of the DSSA pallet extraction simulation ${ }^{3}$. The goal was to verify the AESM by comparison to a similar yet independent simulation. The simulation interval was taken from the start of the pallet (CPSS) extraction to the PTV test article separation trigger. The two simulations compared very well and verification was successful. Figure 2 shows a diagram of the test article and a snapshot of an actual 
extraction from CDT-2. Figure 1 is a series of plots of simulation output from AESM and DSSA. The green DSSA plot trace ends at the planned PTV separation time. The AESM plot trace follows a hypothetical trajectory where the system remains mated past the separation time. Visually, the two predictions are very similar. Successful comparisons with DSS of the PTV in free flight after separation were also performed. Additional comparisons will be performed as the test program continues.

\section{AESM Modeling Principles}

ADAMS is a multi-body dynamic and motion analysis software tool. ADAMS helps engineers to study the dynamics of moving parts, how loads and forces are distributed throughout mechanical systems, and to improve the performance of their systems. The simulation capability was first introduced on the ARES project. It was used to successfully simulate the extraction events for the ARES Parachute Drop Test ${ }^{4}$. The ARES model simulated the extraction, reorientation and release of the Jumbo Drop Test Vehicle (JDTV) from the aircraft and cradle. This modeling effort saved the program money by eliminating the need to redesign the reorientation and release sequence. The next application of the simulation was on CPAS. ADAMS was employed and tailored to model a Parachute Compartment Drop Test Vehicle (PCDTV). Results from this effort gave the CPAS analysis team confidence to apply the cutting edge modeling to a PTV configuration. The PTV test configuration presented a challenge that was less dynamically forgiving compared to a PCDTV. Another challenge posed with testing and modeling a PTV is the fact that the center of mass (cm) and center of pressure (cp) are in close proximity compared to a dart shaped vehicle. The ARES JDTV and CPAS PCDTV are both aerodynamically stable when decelerating on its weighted nose. The PTV simulation objectives are: 1) Determine a point in time to release the PTV from the CPSS, 2) Determine the interactive loads between bodies during extraction/separation, 3) Provide state vector initial conditions to the DSS model, and 4) Ensure a predictable and repeatable separation between the PTV and CPSS.

\section{A. Modeling Components}

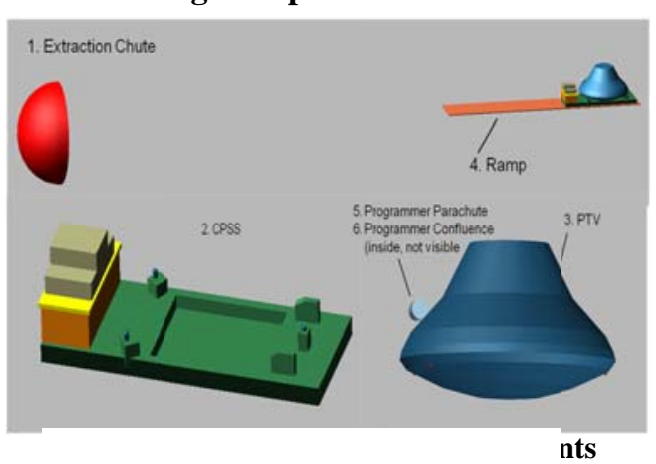

The main components of the AESM are defined by six rigid bodies as depicted in Figure 3.

The interaction and motion of the parts are governed by constraints, enforced motions, external forces, contact forces between bodies and gravity. Every AESM part has six degrees-offreedom (DOF) until the modeler enforces constraints between the bodies. Initially the PTV, CPSS, Programmer, Programmer Confluence and ramp are all constrained relative to each other. The motion of the aircraft ramp is considered an input parameter to this model. The motion statement defines the motion of the aircraft ramp relative to the ground for all 6 DOF, i.e., the airspeed and ramp attitude angles.

The simulation begins as two independent bodies, the extraction parachute and aircraft ramp. At initialization, the aircraft ramp encompasses all the mass properties associated with the mated vehicle. This occurs because the components are constrained relative to one another. The CPSS is fixed to the aircraft ramp and the PTV to the CPSS. As the time-varying simulation executes, predetermined conditions are met, constraints are released and more relative motion between the bodies can take place. For example, the CPSS is fixed in all 6 DOF to the ramp until a $1 / 2 \mathrm{~g}$ load is applied to the CPSS from the extraction line. After the $1 / 2 \mathrm{~g}$ load is sensed, the CPSS is allowed to translate $324 \mathrm{IN}$ along the rail restraints which are installed along the length of the aircraft cargo floor. As the mated vehicle reaches the ramp, the pitch constraint is lifted and pitching motions can take place while gravity holds the CPSS to the ramp. At the end of ramp, the CPSS becomes an independent 6 DOF body free falling under a composite extraction parachute. The PTV remains fixed to the CPSS until the conditions of the "Smart Separation Window" are met and separation of the test vehicles takes place. After separation, the PTV becomes an independent 6 DOF body. The Programmer Parachute remains fixed to the PTV until the distance between the Programmer and the CPSS attach point is greater than the length of the static line, $31 \mathrm{ft}$. After the length of the static line is exceeded, the Programmer is its own 6 DOF body. The Programmer Confluence remains fixed to the Programmer until the distance between the Programmer Confluence and the PTV attach points exceeds the length of the harness legs. 


\section{B. User Defined Forces}

There are three types of user defined forces applied in the AESM. They include a drag force from the programmer and extraction parachute, an external force such as aerodynamic forces, and an internal force such as contact forces between bodies. The following forces have been defined in the AESM.

\section{Extraction and Programmer Parachute Drag Force Modeling}

Inflation modeling of dual extraction parachutes in the AESM is represented by using a composite parachute that has an equivalent drag force. The AESM starts when the extraction parachute bag is pulled into the airstream from the aft end of the aircraft. The cluster of two extraction parachutes are assumed to inflate to full open and a drag area degradation factor is applied to account for any performance loss due to cluster interactions and aircraft wake effects during the extraction phase. The composite drag force is applied to the extraction parachute center of pressure (cp) and has three translational components that act at the cp in the direction opposing the velocity vector. The general equation of the three component extraction parachute drag force vector model is:

$$
F=\bar{q} C_{D} S_{\text {ref }}(t)=1 / 2 \rho V^{2} C_{D} S_{\text {ref }}(t)
$$

The programmer parachute inflation model differs from the extraction parachute drag force vector model

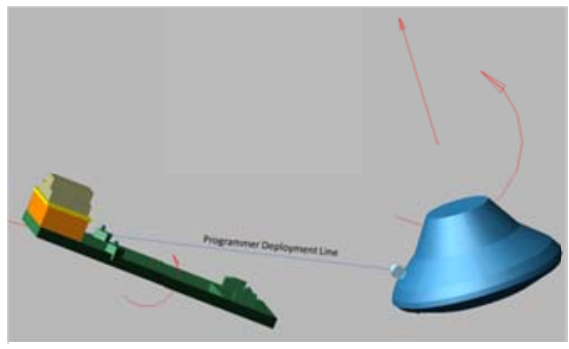
because a time varying inflation curve based on empirical inflation characteristics is applied. Currently, the AESM is only executed and validated through the programmer deployment line stretch event, Figure 4. The programmer phase of flight is in the early development phases and still needs to be anchored to test data before it is officially introduced in the CPAS mainstream simulation sequence. The capability to model independent extraction parachutes using a time varying inflation curve similar to the programmer phase is being integrated for future efforts. This effort would aid in deriving inflation parameters for extraction parachutes

2. Aerodynamic Forces

Aerodynamic forces are applied throughout the duration of the simulation on the mated PTV/CPSS, the CPSS alone and PTV alone. There are a total of six aerodynamic forces and moments acting on the mated vehicle from the end of ramp to the time of separation of the PTV from the CPSS. After separation, the mated aerodynamic forces are deactivated and the CPSS alone and PTV alone aerodynamic forces act on each independent body respectively. The general equation of the aerodynamic force is:

$$
\begin{gathered}
F=\bar{q} C_{x} A_{\text {ref }}=1 / 2 \rho V^{2} C_{x}(\alpha, \beta) A_{\text {ref }} \\
M=\bar{q} C_{x x} A_{\text {ref }} L_{r e f}=1 / 2 \rho V^{2} C_{x x}(\alpha, \beta) A_{\text {ref }} L_{r e f}
\end{gathered}
$$

The mated aerodynamic forces linearly increase from zero at ramp clear to full affect $0.75 \mathrm{~s}-\mathrm{RC}$ later. This is intended to account for the wake effects of the aircraft.

\section{Internal Forces}

Internal forces defined in the AESM fall into two main categories, tension representing lines or slings and contact forces between solid bodies. The lines and slings include: 1) Extraction Line. This line runs from the Extraction Parachute through the single attach point on the CPSS, 2) Four Harness Sling Legs between the PTV attach points
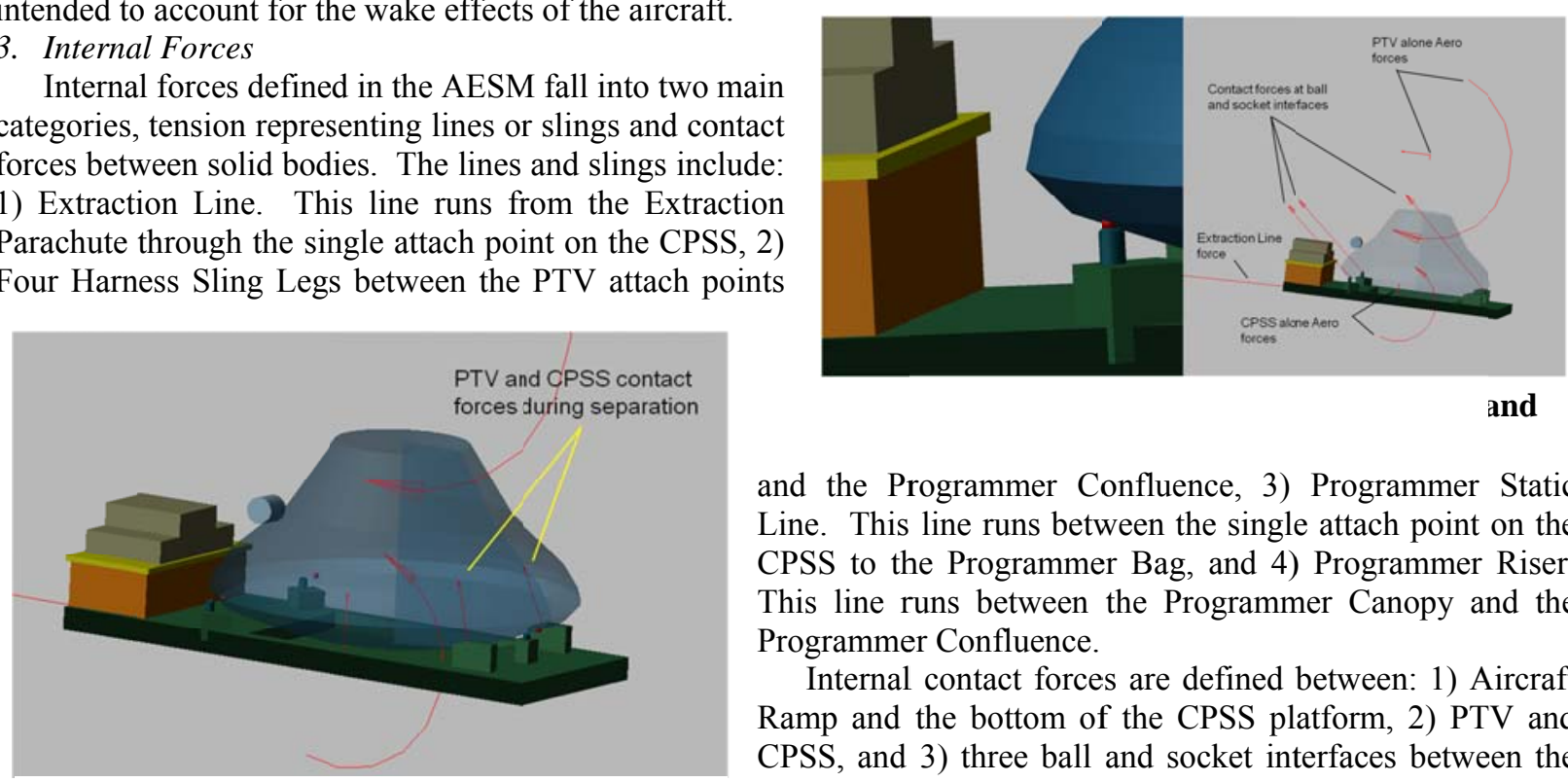

and the Programmer Confluence, 3) Programmer Static Line. This line runs between the single attach point on the CPSS to the Programmer Bag, and 4) Programmer Riser. This line runs between the Programmer Canopy and the Programmer Confluence.

Internal contact forces are defined between: 1) Aircraft Ramp and the bottom of the CPSS platform, 2) PTV and CPSS, and 3) three ball and socket interfaces between the PTV and the CPSS which are part of the release mechanism, Figure 5. The red ball is a rigid member of the 5

American Institute of Aeronautics and Astronautics 
PTV geometry and aqua socket is a rigid member of the CPSS. The most frequent contact between the vehicles occurs between the PTV heat-shield and the front bumpers of the CPSS right after separation as shown in Figure 6.

\section{Atmosphere and Aero Coefficients}

The atmospheric density is required in the calculation used for all aerodynamic and parachute drag forces. This is an input into the AESM via an imported X-Y data table with the density (slugs/ $\left./ \mathrm{ft}^{3}\right)$ as a function of the altitude (ft-MSL). For each drop test, this table has been updated with the local Yuma Proving Grounds (YPG) atmosphere for the month when the test is held.

The six aerodynamic coefficients, Figure 7, are table look-ups handled in a similar manner except they are a function of two independent variables, $\alpha$ and $\beta$, where $\alpha$ is the angle of attack and $\beta$ the side-slip angle. There are 18 aerodynamic effects considered in the simulation, six forces and moments on the mated PTV/CPSS, CPSS alone and PTV alone.

\section{Sensors and Logic Scripts}

AESM is run from a script, or a sequence of commands that allows the program to sense "trigger" conditions, pause and activate/deactivate constraints or external forces and then continue with the simulation. The

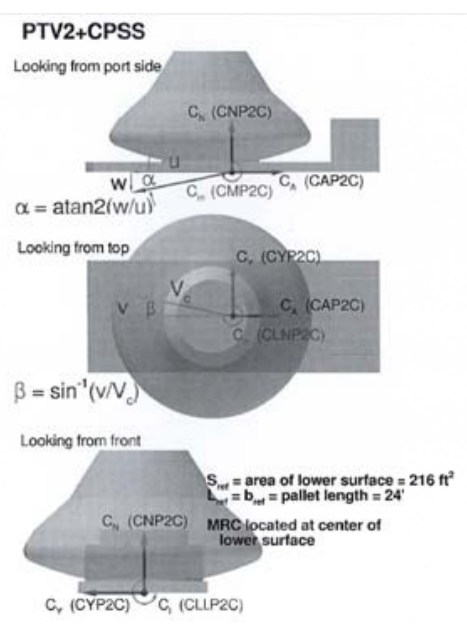
script runs the simulation from the time the extraction parachute is pulled into the airstream until the PTV has fallen under the Programmer parachute 14 seconds. Sensors are set up to define the different triggers and store a "state" such as a time when programmer line stretch occurs so that this time can be referenced later as a constant in the programmer inflation curve equations.

\section{E. Extraction Parachute Center of Pressure}

The extraction parachute is assumed to be a rigid body from the confluence at the bottom of all the suspension lines up around the surface of the canopy. The center of mass $(\mathrm{cm})$ is at the center of the circle forming the canopy skirt. The center of pressure (cp) is 5 inches aft of the center of mass. By separating the distance between the $\mathrm{cp}$ and $\mathrm{cm}$ the extraction parachute would behave similar to that observed from video.
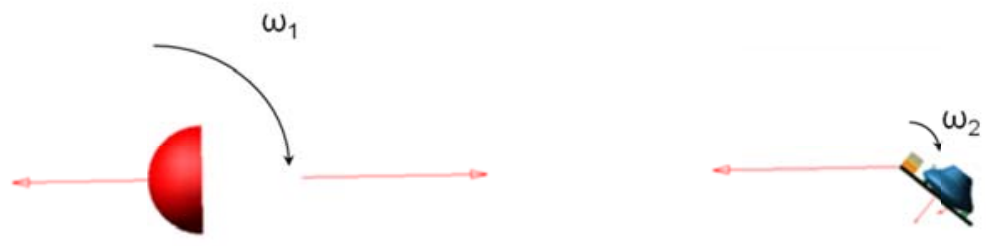

\section{F. Smart Separation Conditions}

The main objective for adopting the AESM was to determine the optimal conditions of releasing the PTV from the CPSS. The general motion of the mated PTV/CPSS after it exits the aircraft is similar to that of a double pendulum. The PTV/CPSS swings about a point near the center of pressure for the extraction parachute and at the attach point of the extraction line and CPSS as depicted on Figure 8.

The timing for the release of the PTV from the CPSS is determined such that: 1) The PTV heat shield will rotate into the wind. This will allow for a clean deployment of the parachutes and reduce the risk of the PTV tangling in the parachute lines, 2) the rotation rate of the PTV

at release is low enough so the PTV will not tumble out of control before the programmer parachute has time to deploy and inflate, and 3) there will be no unplanned impact between the PTV and CPSS to enable the rest of the test to flow smoothly.

An IMU on board the CPSS measures the

\begin{tabular}{|c|c|}
\hline Parameter & Inputs \\
\hline Time $(\mathrm{sec})$ & $1.0<$ time $<4.0$ \\
\hline Pitch Angle $\left(^{\circ}\right)$ & $-25^{\circ} \leq \theta \leq-3^{\circ}$ \\
\hline Pitch Rate $\left({ }^{\circ} / \mathrm{sec}\right)$ & $10^{\circ} / \mathrm{s} \leq \dot{\theta} \leq 25^{\circ} / \mathrm{s}$ \\
\hline
\end{tabular}
acceleration, angular positions and angular rotation rates. The conditions referenced to determine the timing of release are known as the, "Smart Separation Window (SSW)." They included conditions for the pitch attitude, the pitch rate and time from ramp clear. The time variable was put into the SSW parameters to ensure sufficient clearance from the aircraft and, in the event of an IMU failure, release at the end of the time window to give the test 
a chance to proceed and be successful. The AESM simulation helped us determine the conditions of the Smart Separation Window as shown in Error! Reference source not found..

When the separation of the PTV from the CPSS takes place the following sequence of events and interactive forces occur. The aerodynamic forces acting on the test articles change from the mated properties to the independent vehicle properties. The once mated vehicle becomes independent 6 DOF objects, Figure 9. As the mated vehicle

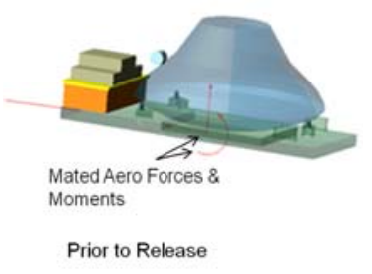

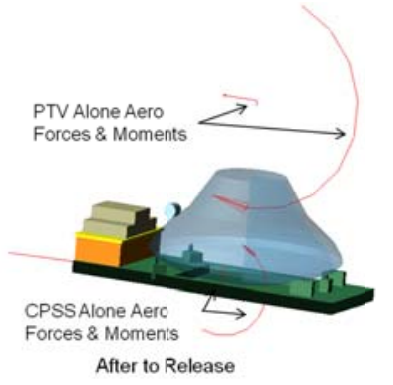

I Separation

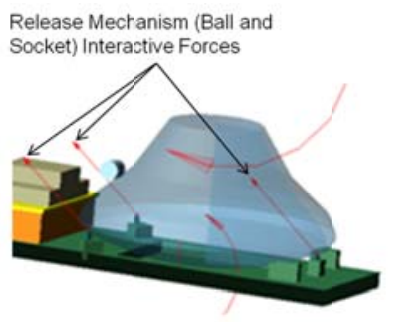

(a)

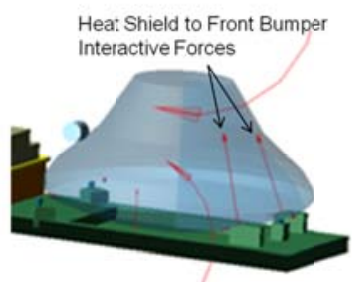

(b)

separates, a level of contact is maintained between the surfaces of the release mechanism in the form of a ball-andsocket joint. The PTV ball slides away from the socket on the CPSS and maintains about the same pitch and pitch rate attitude as the CPSS, Figure 10(a).

Shortly after the ball and socket clear each other, the front bumper slide along the heat shield of the PTV, Figure 10(b). The interaction and aerodynamic effects induce a moment about the PTV $\mathrm{cm}$ to keep the PTV rotating in a heat shield first attitude.

\section{G. Programmer Parachute Implementation}

The Programmer Parachute is stored on the side of the PTV until the distance between the attach points on the CPSS and Programmer deployment bag exceeds the length of the static line connecting the two markers together as seen in Figure 11(a). After the $31 \mathrm{ft}$ static line length has been exceeded, the Programmer becomes its own 6 DOF body. Within the Programmer deployment bag are two masses, one weighing $51.6 \mathrm{lbs}$ representing the mass of the canopy and risers, the second mass $(22 \mathrm{lbs})$ is the Programmer confluence which joins the four harness legs to the single riser. The four harness legs are tension only springs which connect the confluence to the attach points on the PTV. Two of the harness legs are 198.8 inches in length and two are 189.3 inches. When one or more of the harness legs exceed its free length, the confluence is pulled from the deployment bag and becomes an independent body, Figure 11(b). The static line attached between the CPSS and programmer continues to pull on the Programmer until the length of the Programmer riser $(48.8 \mathrm{ft})$ is deployed. This event is known as line stretch Figure

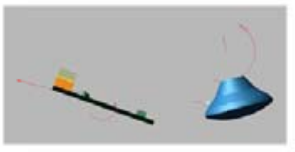

a) Programmer Release

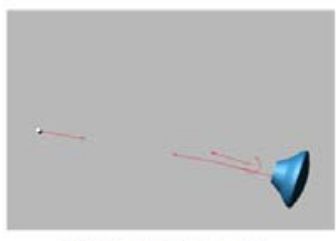

c) Programmer Line Stretch

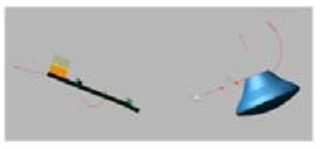

b) Programmer Confluence Release

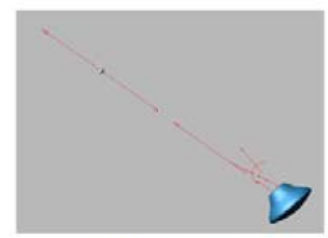

d) Programmer Full Inflation 11(c). Sensors within the AESM store the time of line stretch and velocity of the programmer at line stretch to be used for calculations of the programmer inflation curve in a similar manner as described in the section for extraction parachute inflation.

\section{Data Reconstruction Results}

Post test data reconstructions of the extraction and separation events were performed for EDU-A-CDT-3-3 and EDU-A-CDT-3-5. Each test was unique and did not provide the same separation solution. There were deviations in the extraction parachute performance, force interactions, system event cut triggers, vehicle attitude and day of flight winds which enabled varying downdraft forces. 


\section{A. Avionics Time Delay}

A time delay or time lag observed in measured flight test data was evidently missing compared to the AESM

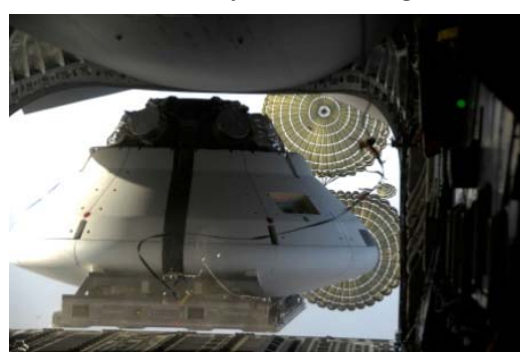

System

Il at RC preflight predictions. The on-board CPSS avionics system includes IMU sensors to measure time, pitch, pitch rate parameters and are activated with a rigged pin-pull mechanism at the ramp clear event as seen in Figure 12. Posttest reconstruction comparisons identified that the avionics system sensed the PTV/CPSS separation criteria on a delay due to processing and executing the cut command to the pyrotechnic cutter system. Another contributor to the observed avionics time delay was attributed to the release mechanism configuration shown in Figure 13 that is used to secure the PTV onto the

\section{CPSS.}

The release mechanism is comprised of a ball and socket geometry which is installed on the PTV and CPSS, respectively. The ball and socket interfaces require additional time to overcome internal interference prior to complete separation. A result of the observed time delays was the implementation of an AESM release mechanism geometry joining the PTV/CPSS vehicles and allowing ball/socket interferences to occur prior to a cleared separation. To account for the known avionics time lag a conservative $90 \mathrm{~ms}$ time delay was included in the smart separation logic to allow for processing time once the conditions were satisfied and execution of the cut command signal.

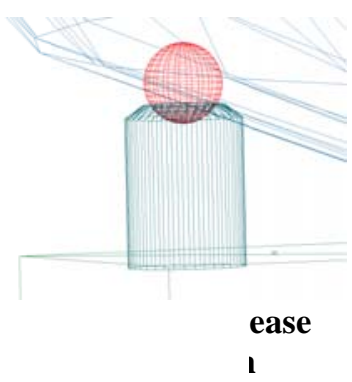

\section{B. Extraction Parachute Inflation Modeling}

A cluster of two $28 \mathrm{ft}$ Extraction Parachutes are simulated as a composite parachute. The initial assumption

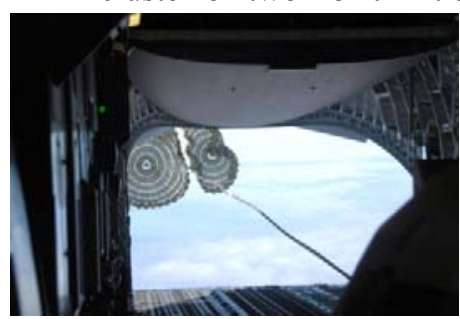
made for modeling the drag force produced by dual extraction parachutes was calculated without consideration of cluster efficiency. However, data reconstruction results confirmed that the extraction parachutes cluster efficiency was reduced $15 \%$ in a cluster of two. The magnitude of the drag force was reduced due to cluster parachute interactions during the inflation process. Each extraction parachute did not inflate to full open during the chaotic extraction phase due to interference and crowding relative to one another. This cluster interaction can be seen in Figure 14. Other factors that contribute to the reduced luster cluster efficiency are the aircraft wake, which is currently not directly quantified.

\section{Extraction Force Line of Action}

The extraction force line of action (EFLA) is the tension that is exerted through the simulated risers and suspension lines of the Extraction Parachutes about the attach point on the mated vehicle. The pre-test orientation of the EFLA was set horizontal to the ground at extraction. Upon further
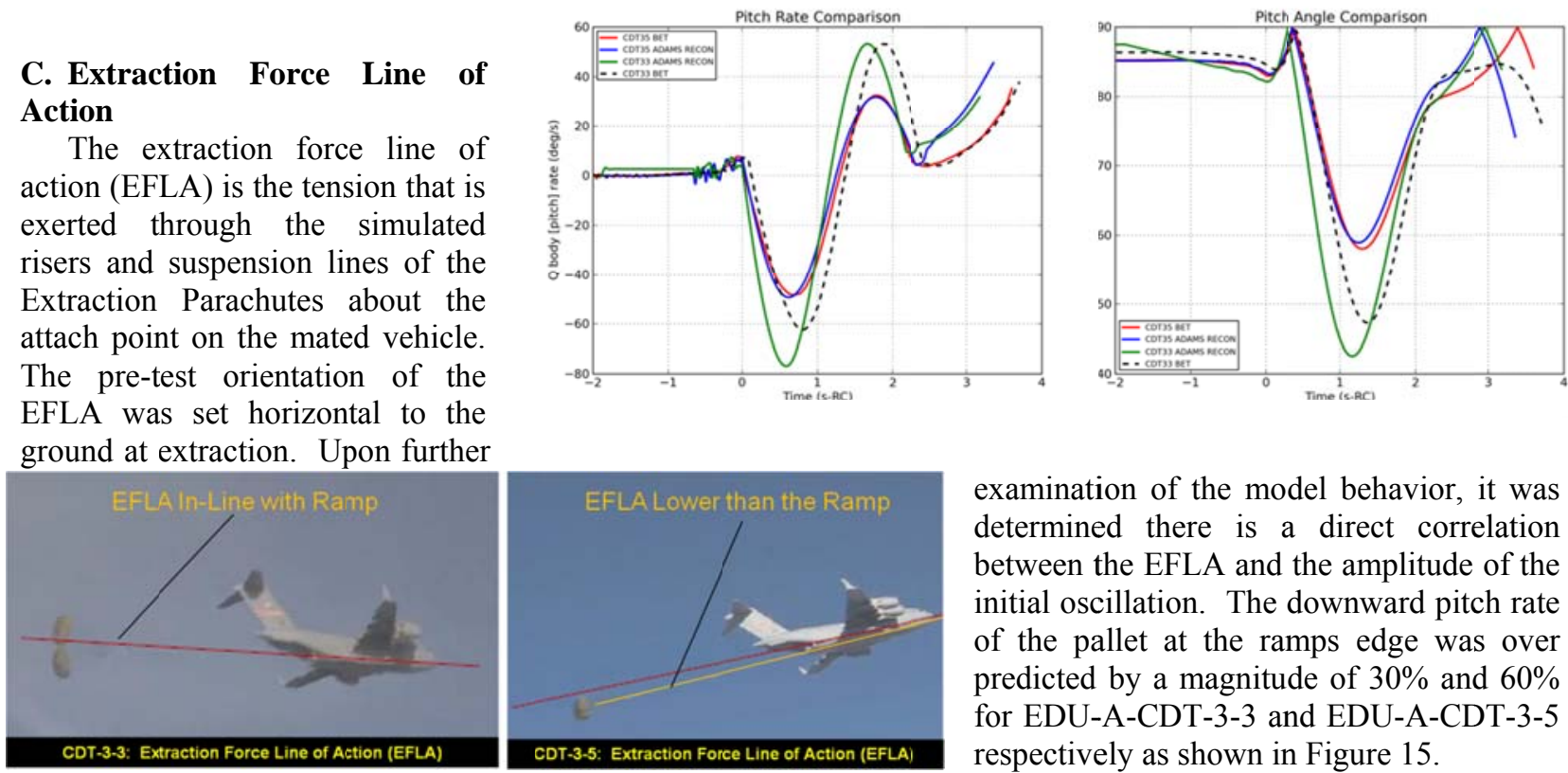

examination of the model behavior, it was determined there is a direct correlation between the EFLA and the amplitude of the initial oscillation. The downward pitch rate of the pallet at the ramps edge was over predicted by a magnitude of $30 \%$ and $60 \%$ for EDU-A-CDT-3-3 and EDU-A-CDT-3-5 respectively as shown in Figure 15.

The post-test video verified the EDU-ACDT-3-5 EFLA appeared oriented lower in

8

American Institute of Aeronautics and Astronautics 
relation with the ramp plane compared to EDU-A-CDT-3-3, Figure 16. In order to vary the position of the extraction parachutes a new variable needed to be introduced in the AESM and was called the downdraft force. As the extraction parachute travels in the wake of the aircraft, there is an airflow that streamlines over the aircraft and converges behind the aircraft in the region of the extraction parachute, driving the extraction parachute further downward than the no wake airflow. The downdraft force is used to control the position of the extraction parachute relative to the ramp plane. It is applied in the simulation as a time step function that is activated 1.2 seconds after the first motion of the mated vehicle on the aircraft ramp. As the PTV/CPSS is extracted, the downdraft force is applied for a one second duration either pushing the extraction parachutes below, in-line, or slightly above the ramp plane. A nominal peak value for the composite drag force is about 39,000 lbs. The equation for the z-component of the extraction parachute drag force is

$$
F=\bar{q} C_{D} S_{r e f}(t)\left(-v_{z} / v_{m}\right)+\text { downdraft }
$$

The downdraft force seen in the two flight tests was $800 \mathrm{lbs}$ and $4100 \mathrm{lbs}$, respectively with the nominal input value set to $2500 \mathrm{lbs}$. Each test had different extraction parachute orientations. The orientation for each test was positioned either over and under or side by side as seen in Figure 16. The $4100 \mathrm{lbs}$ downdraft force used for EDUA-CDT-3-5 produced a good match to the measured test data as shown in Error! Reference source not found.. As the downdraft force increases, the magnitude of the pitch and pitch rate will decrease. For EDU-A-CDT-3-3, the magnitude of the pitch rate was different during the initial descent of the payload, but the overall timing of the PTV release has proven to be exceptional.

\section{Vehicle Dynamics}

Predicting vehicle dynamics and the time sequence of the initial phases of flight has evolved from assuming instantaneous separation of bodies to modeling internal force interactions. The understanding of how to initialize the AESM with representative initial conditions was the first lesson acquired during the post test reconstruction of EDU-A-CDT-3-3. Data comparisons showed that the preflight prediction reached the end of the ramp faster than the actual measured true airspeeds. The slower flight test extraction of the PTV/CPSS was a consequence of contact forces experienced between the CPSS pallet and C-17 ramp. The initial AESM assumed no frictional loss and

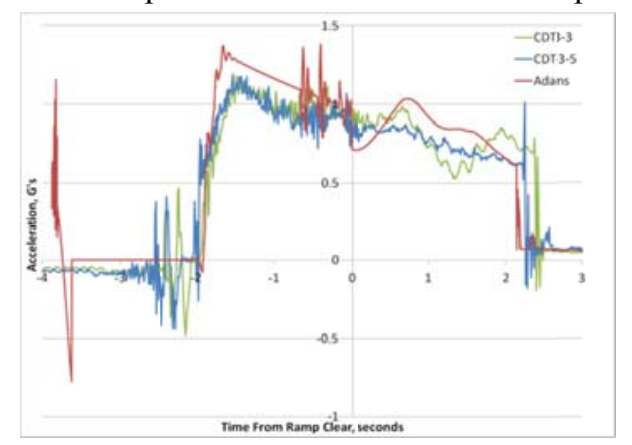
resulted in a faster extraction sequence. The addition of frictional forces between the pallet and ramp improved the extraction timing.

\section{Monte Carlo Analysis}

A Monte Carlo (MC) capability was developed to analyze the unknown trajectory types that could be triggered with a unique set of parameters. To initialize the $\mathrm{MC}$ analysis a representative dispersion band for the aircraft ramp pitch and pitch rate was required. These parameters were defined using a limited test data set acquired from an on-board C-130A and C-17 aircraft sensor tray. C-130A data was included due to limited availability of C-17 only data. An initial conservative dispersion derived from the diverse data points resulted in a $\left[0^{\circ}-10^{\circ}\right]$ pitch and $\left[0^{\circ} / \mathrm{s}-10^{\circ} / \mathrm{s}\right]$ pitch rate band. The dispersion band was intended to cover all the $\mathrm{C}-130 \mathrm{~A}$ and $\mathrm{C}-17$ data experience. This proved to be too conservative and a second approach was implemented to average the aircraft flight test pitch and pitch rate values at ramp clear versus bounding extreme attitudes and rates. This approach delivered a more representative dispersion band of the dynamics that could be seen when extracting from a C-17. The following criteria were used to initialize the aircraft in the AESM at ramp clear. Post test analysis demonstrated that the derived dispersion band captured what was experienced in test by the $\mathrm{C}-17$. The actual C-17 aircraft pitch and pitch rate values seen during EDU-A-CDT-3-5 at ramp clear were $3.5^{\circ}$ and $2.0^{\circ} \mathrm{sec}$, respectively. All other mass property inertias were dispersed $\pm 10 \%$ and the altitude was set to $\pm 500 \mathrm{ft}$.

The MC capability provided insight into trajectory variations and introduced a unique solution that required safeguarding to reduce malfunction risks during the extraction/separation phases of flight. All preflight $\mathrm{MC}$ predictions prior to EDU-AC-17 Aircraft Initialization Parameter Minimum Maximum

\begin{tabular}{|c|c|c|}
\hline Ramp Pitch Angle $\left({ }^{\circ}\right)$ & 0.0 & 5.0 \\
\hline Ramp Pitch Rate $(\% / \mathrm{sec})$ & 0.0 & 5.33 \\
\hline
\end{tabular}

CDT-3-7 only entered the Smart Separation Window (SSW) from the maximum pitch rate side. A MC cycle was considered a satisfactory solution if the PTV pitch rate at separation was above $0^{\circ} / \mathrm{sec}$ and had a $\alpha \geq 95^{\circ}$. This 
requirement helped oppose any apex forward attitude at separation and allowed adequate time for the Programmer parachute to recover control authority of the vehicle and re-orient heat shield forward. The introduction of the down draft force variable transformed how AESM trajectories entered the SSW and aided in identifying three classifications of trajectories. Each trajectory class is defined by the SSW plane it enters: Maximum Pitch Rate (MaxPR), Minimum Pitch Rate (MinPR), and Minimum Pitch Angle (MinPA). The AESM has progressively been anchored to test data and has supported analysis efforts to disclose the physical conditions that produce unfavorable attitudes and identify the possibility of trajectories separating outside the defined SSW using MC analysis.

A new trend of Monte Carlo trajectories was observed with the implementation of the downdraft force and avionics time delay. Trajectories in the right conditions now could potentially enter the non-optimal SSW from the MinPR and MinPA window. The novel trajectory class that entered the MinPR window was attributed with having downdraft forces exceeding $4300 \mathrm{lbs}$ coupled with ramp pitch and ramp pitch rate inputs below $2^{\circ}$ and $1 \% \mathrm{sec}$, respectively. One of the ramp initialization parameters usually had an input below 1 , such as a $0.7^{\circ} / \mathrm{sec}$ pitch rate or $0.4^{\circ}$ pitch.

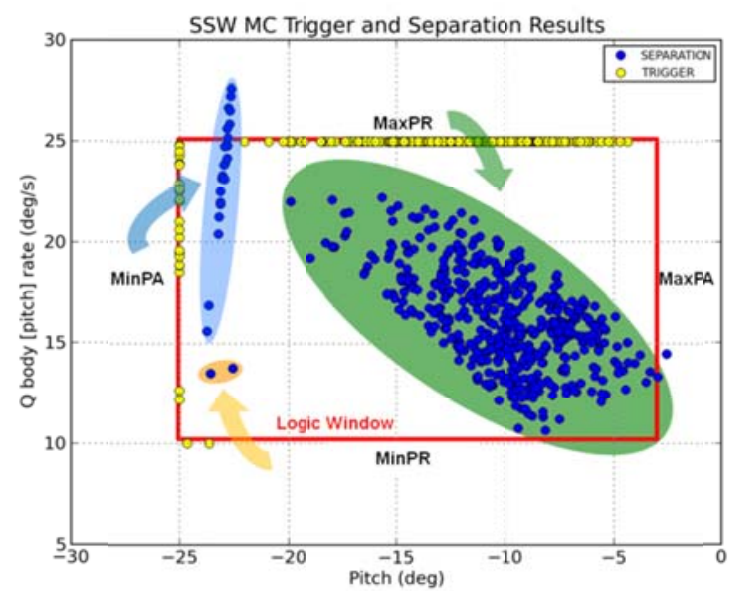

Under these conditions, if the MinPR window was set too low, such as at $0 \% \mathrm{sec}$, the PTV would tend to accelerate apex forward at separation with high risks of snagging lines and tumbling without recovery. An example is shown by the orange case shown in Figure 19. Extreme downdraft forces also produced cases with flat or translational separations. This scenario orients the PTV as it sits on the CPSS with an $\alpha=90^{\circ}$ and slides off with small rates, as if apparently motionless, until the force of the inflating programmer parachute is applied to the PTV at the four attach points.

The second novel trajectory class which entered the MinPA window, like the MinPR class, had large downdraft forces associated with its inputs. The ramp initialization inputs were contributors to entering a different side of the

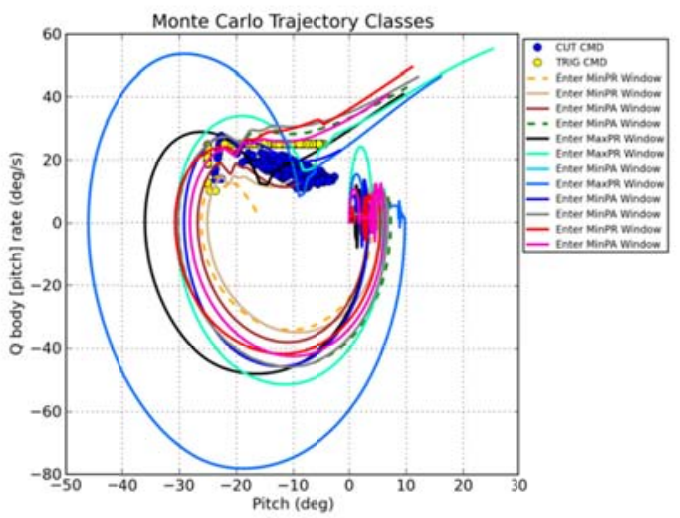

the Smart window. The MinPA ramp inputs were both typically greater than $2^{\circ}$ and $2^{\circ} / \mathrm{sec}$, respectively. The larger ramp initialization values affected the oscillation magnitudes of the pitch and pitch rate at extraction, but still produced heat shield forward PTV attitudes with satisfactory separation results. Cases that entered the MinPA side were also susceptible to triggering within the window limits and executing the separation cut command outside the defined SSW. This behavior was due to the systems positive acceleration and implementation of the $90 \mathrm{~ms}$ avionic system time lag. The SSW conditions were being met prior to reaching the systems peak acceleration and as a result separated and accelerated outside the defined window. Conversely, trajectories entering the MaxPR window satisfy the SSW conditions when the system has already begun to decelerate under the extraction parachutes on the negative slope of the acceleration curve. An understanding of the systems trigger and release cut command timing was acquired and implemented in the AESM. Acquiring an insight to the systems timing sequence eliminated the assumption of instantaneous separation and was replaced with a separation observed in post-test reconstructions. The SSW event was now split into two visible features, a trigger event in which the conditions are sensed and a cut command signal event that is executed a delta time after the system senses the defined separation conditions, Figure 18.

Earlier AESM predictions have modeled higher pitch rates at separation than actually experienced. This was attributed to the delays in the separation event. From when the conditions are sensed to when the actual strap cutters are activated, a period of time has passed resulting in different pitch angle and pitch rate states. A typical rate loss for EDU-A-CDT-3-5 was approximately $10^{\circ} / \mathrm{sec}$. The predicted separation pitch rate was $15 \% \mathrm{sec}$ and the actual test 
data showed we separated at approximately $5 \%$ sec, Figure 20 . The same behavior was seen for all measured angles and rates. Protecting from pitch rate reaching the $0 \% \mathrm{sec}$ threshold has prompted analysts to raise the MinPR window to $10 \%$ sec to safeguard from unfavorable trajectories that enter from the MaxPR and MinPR window. The enhancement of the AESM and MC capability has resulted in flight-like preflight predictions. The system performance for the extraction and separation phase of flight can now be correlated to test data and simulate the pitch and pitch rate seen in flight tests at separation.

\section{Conclusion}

The development and integration of the AESM into the CPAS mainstream simulation sequence has introduced state-of-the-art extraction/separation analysis techniques and improved the fidelity of state vectors required to initiate primary NASA driven simulations. The assumption of modeling instantaneous separation of CPAS test articles has been replaced with a new convention that accounts for $90 \mathrm{~ms}$ avionics system time delays, internal force interactions, and downdraft forces. These have been adopted with exceptional test results.

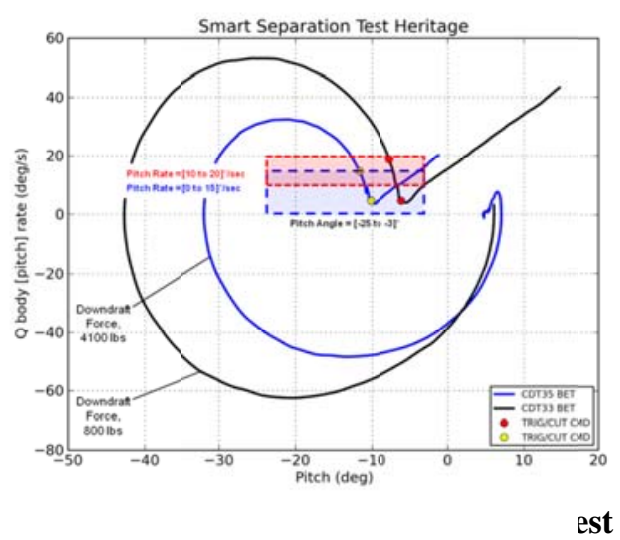

Comparisons alongside established NASA simulations and test data reconstruction efforts have been performed to verify and validate the AESM. A physics-based solution to the PTV/CPSS extraction-separation event has been established with the insight attained of the force interactions and recontact phenomenon that occur during release. The moment-forces generated by the extraction parachute and mated vehicle during the free fall flight phase are better understood and can now be harnessed with a smart separation

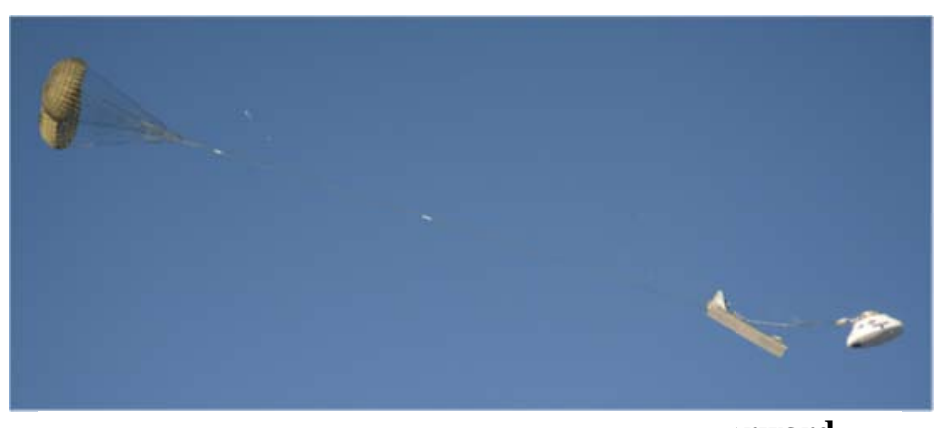
window for optimal PTV heat-shield forward attitudes. Interferences between the PTV heatshield and CPSS front bumpers support favorable attitudes by opposing aerodynamic moments that tend to orient the PTV apex forward.

The addition of the downdraft force has enhanced our thinking and shed light on two new trajectory classes that enter the smart separation window. As a result, the MinPR window limit was shifted from $0 \% \mathrm{sec}$ to orward $10 \% \mathrm{sec}$ to protect from PTV cases with negative pitch rates and $\alpha<95^{\circ}$ that result in apex forward attitudes at programmer deployment. Earlier defined windows assumed that any non-negative pitch rate would result in favorable PTV attitude. Cases entering the MinPA window demonstrated cases accelerating outside the defined window. These cases were attributed to downdraft forces above $4100 \mathrm{lbs}$. The overall fundamental understanding of the vehicle dynamics experienced during the initial phases of flight has improved. This allowed CPAS to overcome challenges in this highly complex test sequence. 


\section{References}

${ }^{1}$ Machin, Ricardo A. and Evans, Carol T., "Cluster Development Test 2 an Assessment of a Failed Test," $20^{\text {th }}$ AIAA Aerodynamic Decelerator Systems Technology Conference and Seminar, Seattle, Washington, 4 - 7 May 2009, AIAA paper 2009-2902.

${ }^{2}$ Cuthbert, P.A. and Desabrais, K.J., "Validation of a Cargo Airdrop Software Simulator," 17th AIAA Aerodynamic Decelerator Systems Technology Conference, Monterey, California, May 20-22, 2003, AIAA Paper 2003-2133.

${ }^{3}$ Cuthbert, P.A., "A Software Simulation of Cargo Drop Tests," 17th AIAA Aerodynamic Decelerator Systems Technology Conference, Monterey, California, May 2003, AIAA Paper 2003-2132.

${ }^{4}$ King, Ronald, Wolf, Dean, and Hengel, John., "Ares Decelerator System Heavy Air Drop Test Program," 21st AIAA Aerodynamic Decelerator Systems Technology Conference, Dublin, Ireland, March 2011, AIAA Paper 2011-2504.

${ }^{5}$ Stuart, Philip,"CPAS Aerodynamic Database Application Programming Interface User's Guide," Version 2.0.0b2, CPAS Analysis IPT, NASA-JSC EG Document, December 2011. 